\title{
Definição e Gerência de Objetivos de Software Alinhados ao Planejamento Estratégico
}

\author{
Andrea Oliveira Soares Barreto, Ana Regina Cavalcanti da Rocha
}

COPPE/UFRJ - Universidade Federal do Rio de Janeiro

Caixa Postal 68511 - CEP 21945-970 - Rio de Janeiro, Brazil

\{ansoares, darocha\}@cos.ufrj.br

\begin{abstract}
This paper presents an approach to support the definition of the business aligned software improvement goals and also to support the management of these goals. The approach promotes continuous monitoring of the defined goals and the execution of appropriate actions to address detected deviation, considering also the scenario of high maturity software organizations. This approach comprises three main components: a Method for Strategic, Tactical and Operational Planning in software organizations; an Infrastructure for Monitoring Strategic, Tactical and Operational Goals; and a Strategy for Corrective Actions Recommendation. The approach development and evaluation and the main results are described in this paper.
\end{abstract}

Resumo. Este trabalho apresenta uma abordagem que apoia a definição de objetivos de software alinhados ao negócio da organização e a gerência desses objetivos. A abordagem promove a monitoração contínua dos objetivos definidos e a execução de ações apropriadas para tratar os desvios detectados, considerando, inclusive o cenário das organizações de software de alta maturidade. Essa abordagem contém três componentes principais: um Método para Planejamento Estratégico, Tático e Operacional em organizações de software; uma Infraestrutura para Monitoração dos Objetivos definidos; e uma Estratégia para Recomendação de Ações Corretivas. $O$ desenvolvimento da abordagem, sua avaliação e os principais resultados obtidos são descritos neste artigo.

\section{Introdução}

Organizações que buscam a melhoria de seus processos de software precisam se preocupar em implementar melhorias que estejam alinhadas ao negócio da organização. Estudos indicam que o alinhamento dessa melhoria aos objetivos de negócio é um dos fatores críticos de sucesso (Pries-Heje e Johansen 2010; Guzmán et al. 2010; Montoni 2010). Assim, ao identificar seus objetivos de melhoria de software, uma organização precisa se preocupar em definir objetivos alinhados aos objetivos de negócio. Neste trabalho é utilizado o conceito de "objetivo de software" como um tipo de objetivo de melhoria de software que: é uma decomposição de um objetivo de negócio; é mensurável; e diz respeito apenas a produtos ou processos de software (Barreto 2011).

Apesar de existirem na literatura várias evidências da necessidade de alinhamento das melhorias de processos de software aos objetivos de negócio obter esse alinhamento pode não ser tão simples (Becker et al. 2008). Especificamente em 
organizações de alta maturidade, obter esse alinhamento pode se mostrar mais complexo, uma vez que, neste contexto, a organização precisa, ainda, planejar o controle estatístico de seus processos de software (SEI 2010; SOFTEX 2009).

Além de definir objetivos de melhoria de software alinhados aos seus objetivos de negócio, uma organização também precisa acompanhá-los continuamente de modo a detectar possíveis ameaças ao alcance desses objetivos e, se necessário, tomar ações adequadas com o intuito de facilitar o alcance dos objetivos. Para que essa monitoração seja bem sucedida, um dos problemas a ser tratado, é a dificuldade em detectar desvios, descobrir as causas e então tomar ações apropriadas (Boffoli et al. 2008).

Diante das dificuldades supracitadas e da carência de estudos que tratem essas dificuldades, este trabalho teve como objetivo geral definir uma abordagem que apoie as organizações na definição de objetivos de software alinhados ao negócio da organização e na gerência desses objetivos promovendo a monitoração contínua e a execução de ações mais adequadas para tratar os desvios detectados.

Visando alcançar o objetivo geral deste trabalho e com base nas etapas de um trabalho científico propostas por Wazlawick (2009) e Appolinario (2006), o desenvolvimento da pesquisa foi orientado pelas seguintes etapas: (i) Definição do tema da pesquisa; (ii) Revisão da literatura; (iii) Definição do objetivo da pesquisa; (iv) Elaboração da abordagem proposta; (v) Análise da viabilidade da abordagem proposta; e (vi) Evolução da abordagem proposta. Inicialmente as três primeiras etapas foram executadas de forma iterativa. Posteriormente, a revisão da literatura continuou até a conclusão do trabalho, inclusive com a execução de estudos baseados em revisão sistemática da literatura. A partir do objetivo definido, iniciou-se então a elaboração da abordagem proposta, que é composta por três componentes principais, conforme detalhado na seção 4. Nessa etapa os componentes da abordagem foram identificados, a proposta para cada componente foi elaborada e as ferramentas de apoio à utilização da abordagem foram desenvolvidas. Para apoiar a elaboração da proposta, um survey foi realizado, conforme descrito na seção 4.3. Para cada componente da abordagem, à medida que a etapa de elaboração da proposta foi concluída, um estudo de viabilidade foi realizado. O primeiro e o segundo componentes foram utilizados em organizações de software, e, para o terceiro componente, a viabilidade foi analisada com base em um estudo experimental, conforme descrito na seção 5. Para apoiar o planejamento e a execução do estudo experimental, dois estudos baseados em revisão sistemática da literatura foram realizados, conforme descreve a seção 5. Por fim, a partir dessas análises de viabilidade, a abordagem proposta inicialmente foi evoluída.

A seção a seguir apresenta uma breve revisão da literatura sobre melhoria de processos de software e planejamento estratégico. A seção 3 descreve algumas abordagens relacionadas à monitoração de objetivos e à execução de ações para tratar eventuais desvios. Na seção 4, a abordagem proposta é descrita em detalhes. A seção 5 descreve as avaliações da abordagem proposta que foram realizadas neste trabalho e a seção 6 apresenta as considerações finais e conclusões deste trabalho.

\section{Alinhamento Estratégico da Melhoria de Processos de Software}

Uma organização pode ser analisada sob três níveis: Estratégico, Tático e Operacional. No nível estratégico são definidos os objetivos estratégicos, que são de longo prazo, e as 
ações para alcançá-los. No nível tático, que é responsável pela comunicação entre os níveis estratégico e operacional, são definidos os objetivos táticos, que normalmente são de médio ou curto prazo, e as ações para alcançá-los. O nível operacional é a base da organização onde são definidas as tarefas a serem executadas (Chiavenato 2000).

No nível tático, no contexto das organizações de software, os objetivos táticos podem ou não estar relacionados ao software. Quando relacionados ao software, pode-se dizer que esses objetivos representam os objetivos de melhoria de software da organização. Nesse nível, além de definirem seus objetivos táticos, organizações de software precisam planejar a medição de software e, se desejam alcançar uma maior maturidade de seus processos de software, elas também precisam planejar o controle estatístico de processos (SEI 2010; SOFTEX 2009).

Alguns trabalhos têm sido desenvolvidos com o objetivo de promover o alinhamento estratégico da melhoria de software, dentre eles: o BOQM - Balanced Objective-Quantifiers Methodology (Guzmán et al. 2010); um roadmap para alinhar a estratégia de negócio e as melhorias de processo de software (Asato et al. 2009); e, uma proposta para a condução de programas de melhoria de processos alinhado aos objetivos organizacionais (Becker 2007). No entanto, observou-se que ainda existiam questões a serem respondidas relacionadas à definição dos objetivos táticos e objetivos de melhoria alinhados estrategicamente, e à integração do planejamento da medição e do controle estatístico de processos ao planejamento tático da organização.

\section{Monitoração de Objetivos e Execução de Ações Corretivas}

Além de definir seus objetivos, uma organização precisa acompanhá-los continuamente, verificar se estão sendo atingidos e detectar previamente desvios que ameacem o alcance desses objetivos. Nesse contexto, a organização precisa tomar ações que a levem a alcançar esses objetivos (McBride 2008). Porém, analisar desvios e determinar quais ações tendem a ser mais adequadas pode ser difícil (Barreto e Rocha 2010a).

Diante dessa dificuldade, sistemas de recomendação têm sido desenvolvidos para auxiliar na busca por informações e apoiar a tomada de decisões em situações onde falta experiência ou não é possível, sem auxílio tecnológico, considerar todas as informações disponíveis (Robillard et al. 2010). Alguns sistemas de recomendação são descritos em (Thies e Roth 2010; Trindade 2009).

Recomendações podem ser baseadas em conhecimento adquirido com experiências anteriores. Uma das abordagens para apoiar a captura, organização e armazenamento de experiências anteriores e o aprendizado sobre como resolver novos problemas a partir de soluções passadas é o Raciocínio Baseado em Casos - RBC (Case-Based Reasoning - CBR). Nessa abordagem, a análise de similaridade é um fator crítico uma vez que a eficácia do RBC depende essencialmente da escolha de um conceito de similaridade adequado para o domínio da aplicação e a estrutura dos casos usados (Wangenheim e Wangenheim 2003). Algumas aplicações do RBC para recomendação são descritas em (Santos e Cortés 2010; Bomfim et al. 2007).

\section{Abordagem Proposta para Definição e Gerência de Objetivos de Software}

A abordagem proposta neste trabalho é composta por três componentes: (i) Método para

Planejamento Estratégico, Tático e Operacional em Organizações de Software; (ii) 
Infraestrutura para Monitoração de Objetivos; e (iii) Estratégia para Recomendação de Ações Corretivas, conforme mostra a Figura 1.

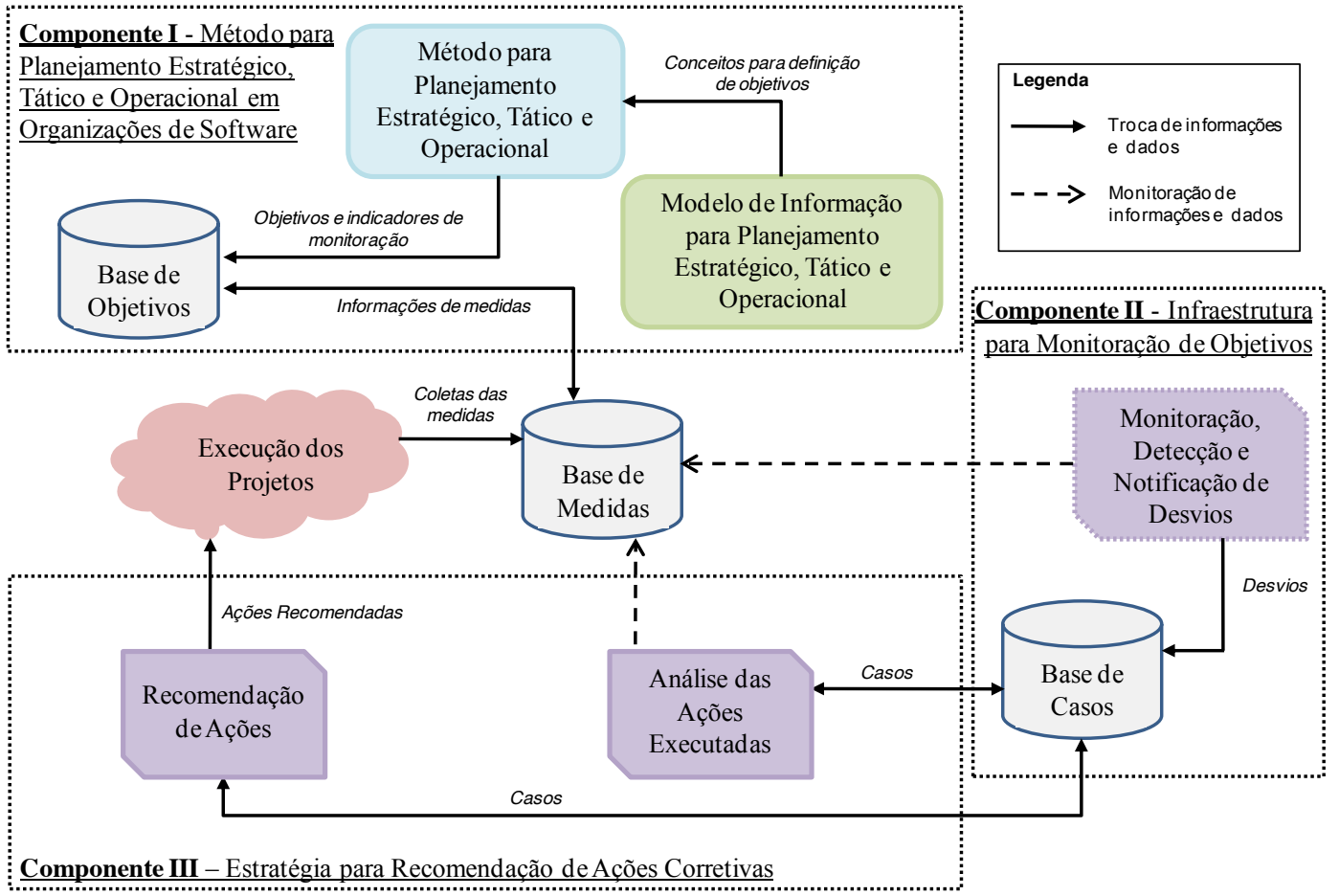

Figura 1. Visão geral da abordagem proposta

A definição e a utilização do método para planejamento estratégico, tático e operacional são apoiadas por um modelo de informação que descreve os conceitos necessários para a elaboração do planejamento nos três níveis. Esse modelo de informação e o método para planejamento estratégico, tático e operacional são usados como base para os demais componentes da abordagem proposta.

A aplicação do método permite a definição de objetivos estratégicos, táticos e operacionais, bem como a associação de indicadores para a monitoração dos objetivos definidos. Esses objetivos são acompanhados pela infraestrutura de monitoração, com base nas medidas coletadas para os indicadores de monitoração. A infraestrutura de monitoração é responsável por analisar continuamente os valores coletados para os indicadores, detectar e notificar a ocorrência de desvios e armazenar os desvios detectados na base de casos que está descrita na seção 4.3.

Durante a notificação de um desvio, a estratégia para recomendação de ações corretivas identifica casos de desvios similares ao desvio atual e recomenda as ações executadas com sucesso nos casos similares. Tanto as ações recomendadas como a ação executada pelo usuário são armazenadas na base de casos. Após a recomendação da ação, a estratégia analisa as novas medidas coletadas para o indicador que evidenciou o desvio e infere o efeito da ação executada. O efeito percebido pela estratégia é submetido à validação do usuário e, então, ele é armazenado na base de casos.

Essa abordagem foi definida considerando a sua aplicação em dois cenários: (i) Cenário sem alta maturidade: cenário das organizações de software cujos processos são 
aderentes aos níveis iniciais de maturidade e que não estão buscando atingir a alta maturidade; e, (ii) Cenário com alta maturidade: cenário das organizações de software cujos processos já são aderentes aos níveis mais altos de maturidade ou que estão buscando atingir a alta maturidade. A principal diferença na aplicação da abordagem proposta nesses cenários está na utilização do método para planejamento estratégico, tático e operacional. No cenário (i), a aplicação do método não precisa considerar o controle estatístico de processos. Assim, os objetivos e indicadores de monitoração são definidos com base na análise de dados de medições anteriores, sem a aplicação de técnicas estatísticas. A aplicação do método no cenário (ii) deve considerar o controle estatístico de processos e a definição de objetivos específicos relacionados aos processos a serem controlados estatisticamente. Nesse cenário, a infraestrutura de monitoração dos objetivos e a estratégia para recomendação também devem monitorar os objetivos relacionados aos processos usando o controle estatístico de processos.

No contexto deste trabalho também foram desenvolvidas as seguintes ferramentas de apoio: (i) Ferramenta de apoio à execução do método para planejamento estratégico, tático e operacional em organizações de software; (ii) Ambiente de apoio à monitoração dos objetivos que implementa a infraestrutura para monitoração de objetivos proposta; e, (iii) Sistema de recomendação de ações corretivas que implementa a estratégia para recomendação de ações corretivas.

\subsection{Método para Planejamento Estratégico, Tático e Operacional}

O método proposto descreve os passos a serem executados em cada um dos níveis e diretrizes para o planejamento. A Figura 2 apresenta a visão geral do método.

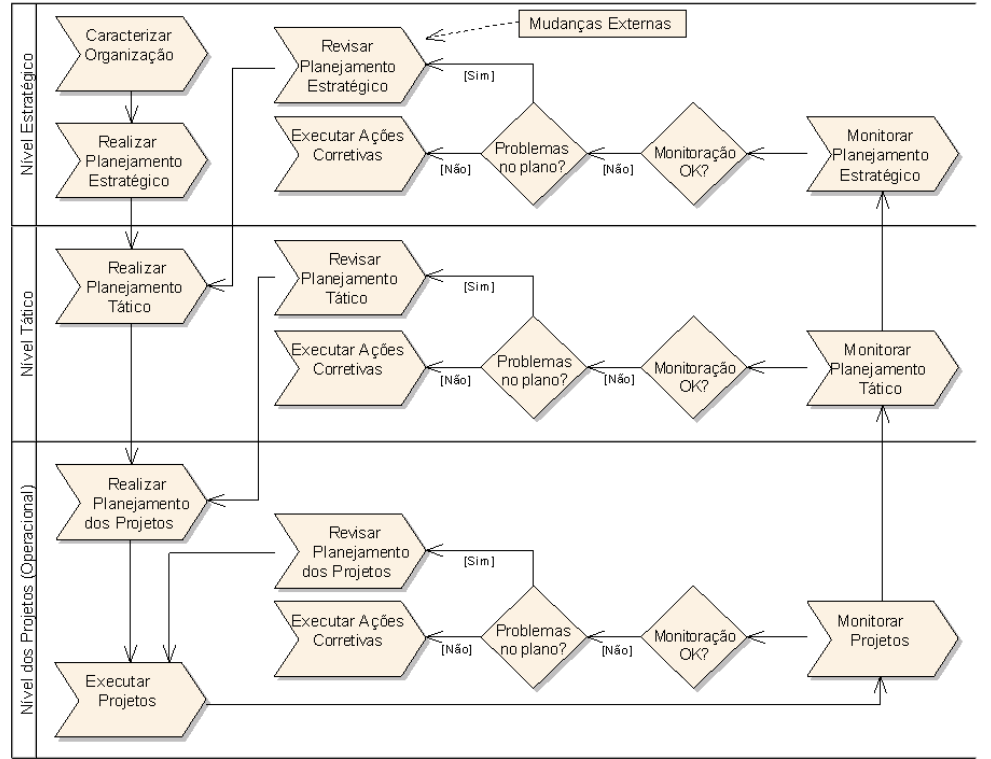

Figura 2. Visão geral do método para planejamento estratégico, tático e operacional em organizações de software

Em cada nível da organização é realizado um planejamento onde os objetivos daquele nível são definidos, juntamente com os indicadores para monitoração de cada objetivo. Os objetivos são monitorados e ações corretivas são executadas, se necessário. 
Para facilitar a elaboração dos planejamentos estratégico, tático e operacional é importante definir alguns conceitos, bem como a relação existente entre eles. Para isso, um modelo de informação para planejamento estratégico, tático e operacional em organizações de software foi proposto neste trabalho. $O$ modelo define uma terminologia padrão e uma relação entre os objetivos, sendo responsável por associar as informações necessárias para definir e monitorar os objetivos estratégicos, táticos e operacionais em organizações de software. Esse modelo descreve os seguintes conceitos básicos: Objetivo Estratégico, Interação entre Objetivos, Iniciativa Estratégica, Objetivo Tático, Objetivo Tático não Relacionado a Software, Objetivo de Software, Objetivo de Software de Médio Prazo, Objetivo de Software de Curto Prazo, Objetivo Quantitativo de Qualidade e Desempenho, Objetivo do Projeto e Indicador de Monitoração.

A Figura 3, a Figura 4 e a Figura 5 apresentam, respectivamente, as etapas para planejamento estratégico, tático e operacional, segundo o método proposto. Maiores detalhes relacionados a cada nível estão descritos em (Barreto e Rocha 2010b).
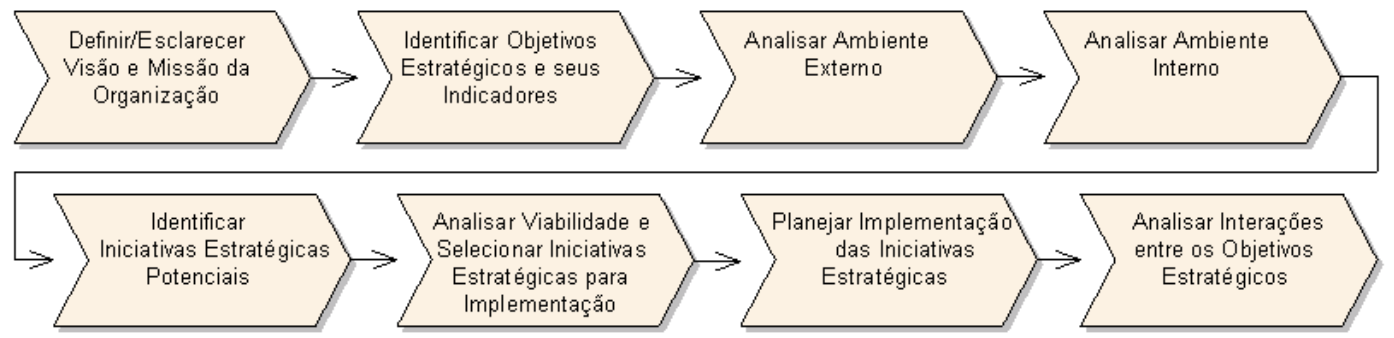

Figura 3. Etapas do planejamento estratégico

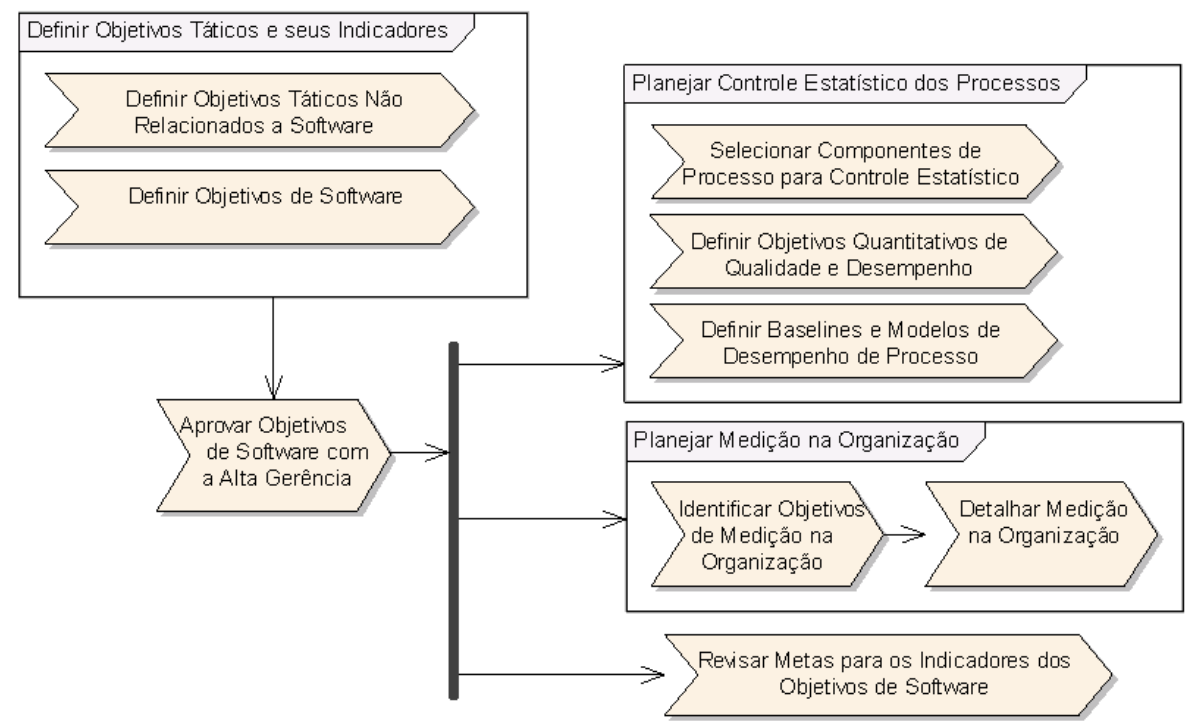

Figura 4. Etapas do planejamento tático 


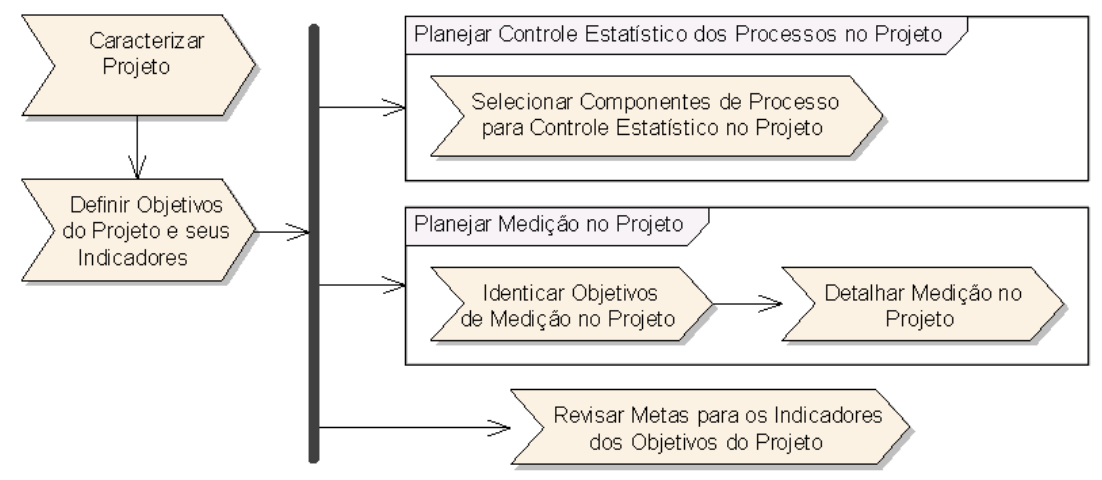

Figura 5. Etapas do planejamento do projeto

\subsection{Infraestrutura para Monitoração de Objetivos}

A infraestrutura para monitoração dos objetivos proposta visa acompanhar continuamente os indicadores associados aos objetivos com o intuito de detectar e notificar eventuais ocorrências de desvios. A Figura 6 ilustra a infraestrutura proposta.

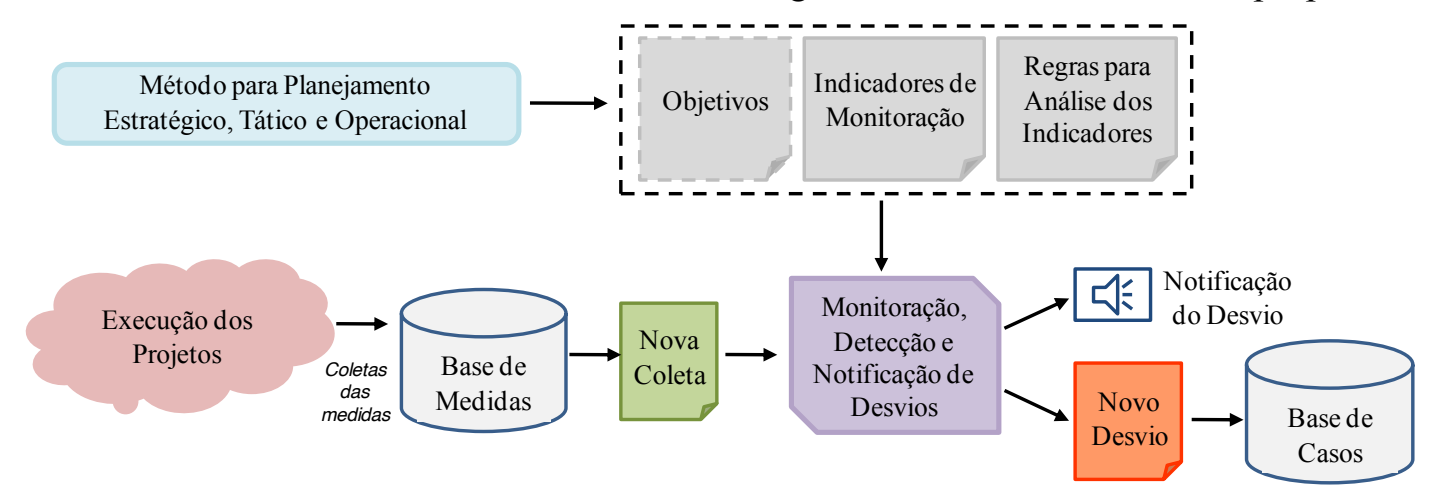

Figura 6. Infraestrutura para Monitoração dos Objetivos

Durante a execução dos projetos, as medidas identificadas no planejamento da medição são coletadas e armazenadas na base de medidas. A infraestrutura acompanha continuamente todas as medidas coletadas na organização, verifica se a medida que está sendo coletada é usada para monitorar algum objetivo e, caso afirmativo, analisa o valor coletado de modo a detectar desvios potenciais ou desvios reais. A análise das medidas coletadas e a detecção dos desvios são baseadas nas regras para análise dos indicadores de monitoração (critérios de decisão) detalhadas juntamente com a definição dos objetivos e indicadores. Para cada coleta registrada que está associada a uma medida usada para monitorar algum objetivo, cada regra definida para análise do indicador, descrita no procedimento de análise associado ao objetivo, é verificada de modo a identificar aquela que é atendida pelo valor coletado. A partir disso, se a regra atendida não está associada a um desvio, a análise da coleta é finalizada e o valor coletado é interpretado como aceitável. Por outro lado, se a regra atendida está associada a um desvio, ele é detectado, armazenado na base de casos, descrita na seção 4.3, e então notificado. A Figura 7 apresenta uma tela do ambiente de apoio à monitoração dos objetivos implementado neste trabalho, destacando a notificação de dois desvios detectados. 


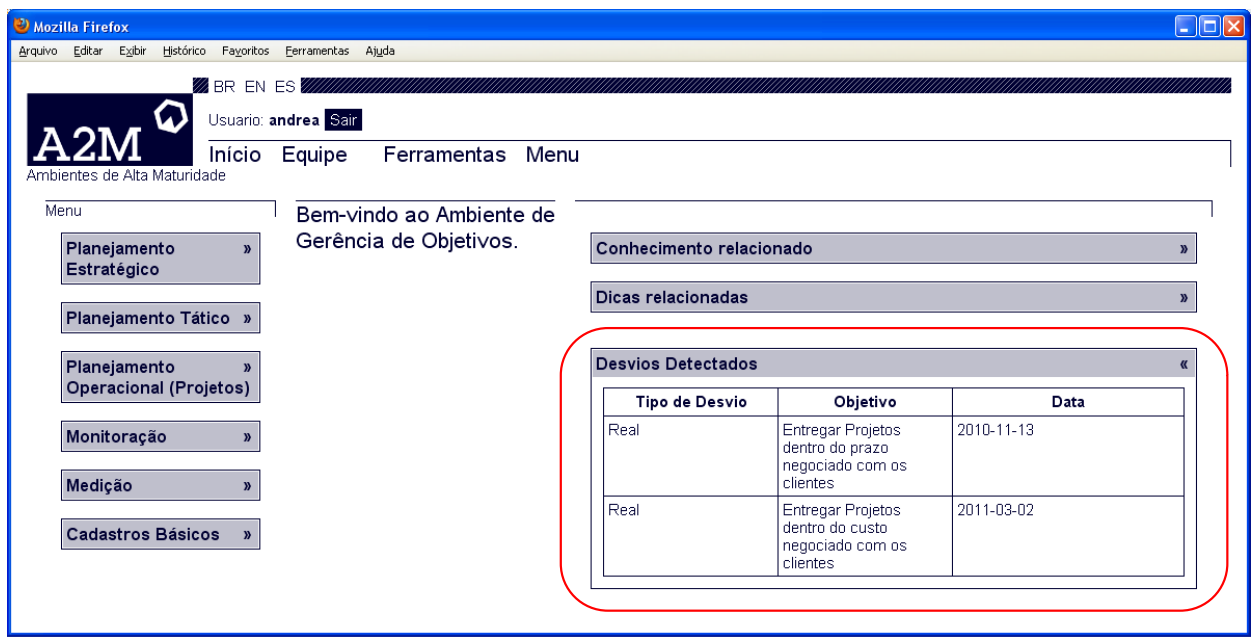

Figura 7. Notificação de desvios no ambiente de apoio à monitoração

\subsection{Estratégia para Recomendação de Ações Corretivas}

Para desenvolver a estratégia para recomendação de ações corretivas proposta, inicialmente o problema a ser tratado pela estratégia foi definido e, então, buscou-se definir uma solução para esse problema, considerando a necessidade de aproveitar o conhecimento associado às experiências anteriores. A partir da solução definida, as etapas para o desenvolvimento da solução foram identificadas e executadas, originando a estratégia proposta. A Figura 8 ilustra essa estratégia.

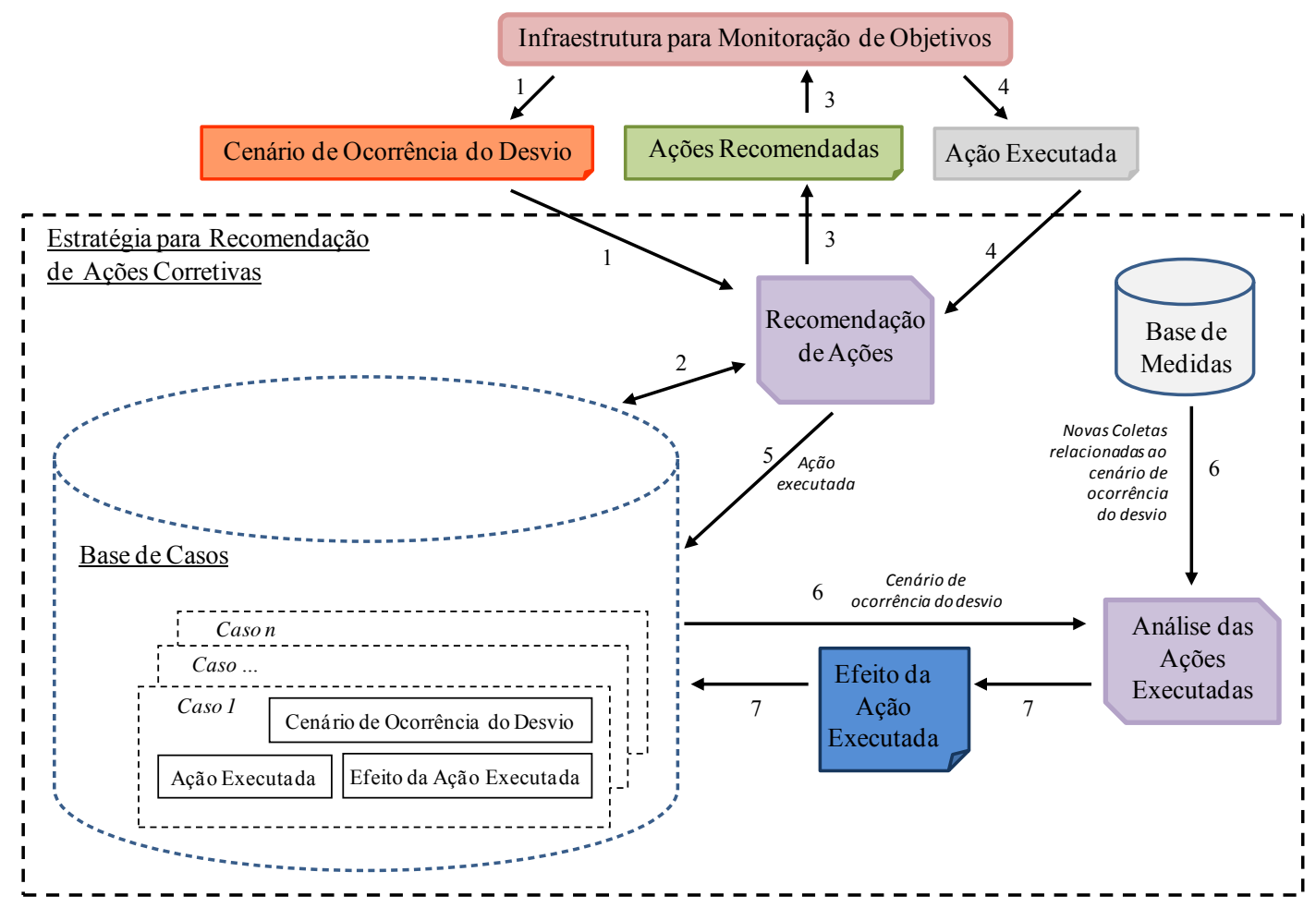

Figura 8. Estratégia para Recomendação de Ações Corretivas 
Essa estratégia aplica o RBC, considerando que um caso contém três partes: problema, solução e avaliação da solução. O problema é descrito como o cenário de ocorrência do desvio. A solução registra a ação executada para tratar o desvio e a avaliação da solução descreve o efeito da ação. O cenário de ocorrência do desvio descreve: informações do desvio, objetivo afetado, informações da coleta do indicador que sinalizou o desvio e contexto da coleta da medida.

Ao detectar um desvio, a infraestrutura para monitoração solicita à estratégia as ações mais adequadas, informando o cenário de ocorrência do desvio. Com base nesse cenário, e considerando os casos armazenados na base de casos, a estratégia para recomendação sugere as ações mais adequadas. Vale destacar que a recomendação das ações mais adequadas envolve sugerir: (i) ações que se mostraram efetivas para tratar os desvios detectados em experiências de desvios que ocorreram anteriormente em cenários similares ao atual e que, por isso, poderiam ser executadas para tratar o desvio atual; e, (ii) ações que não deveriam ser executadas no cenário atual, considerando que em experiências anteriores similares, a execução dessas ações aumentou o desvio, ao invés de tratá-lo. A recomendação da estratégia é apresentada ao usuário que escolhe qual ação será executada, podendo ser qualquer ação, inclusive alguma que não tenha sido recomendada. A ação escolhida para execução é informada à estratégia e esta armazena a ação escolhida na base de casos.

Posteriormente, quando novas coletas são registradas para o indicador que sinalizou o desvio, a estratégia analisa as novas coletas considerando o cenário de ocorrência do desvio e as regras de análise associadas ao indicador que sinalizou o desvio e infere o efeito da ação executada segundo um conjunto de heurísticas definidas neste trabalho, que pode ser positivo, negativo ou indiferente. A análise do efeito realizada pela estratégia é submetida à validação do usuário e então o resultado dessa validação é considerado pela estratégia, ao sugerir as ações mais adequadas.

Uma das definições mais importantes para a análise de similaridade entre casos é a definição de quais informações serão consideradas na comparação entre casos, isto é, os índices do caso. Buscando-se caracterizar um conjunto de informações relevantes para indicar o grau de similaridade entre cenários de ocorrência de desvios, um survey foi realizado. Como principal resultado, foi obtido um conjunto de características de projeto e o grau de relevância de cada característica para a análise de similaridade entre cenários de ocorrência de desvios, conforme mostra a Tabela 1. Uma descrição detalhada do planejamento e execução do survey, bem como a análise dos resultados obtidos estão descritas em (Barreto e Rocha 2010a).

Tabela 1. Conjunto de características resultante do survey

\begin{tabular}{|c|c|c|c|c|c|}
\hline Característica & $\begin{array}{c}\text { Grau de } \\
\text { Relevância }\end{array}$ & Característica & $\begin{array}{c}\text { Grau de } \\
\text { Relevância }\end{array}$ & Característica & $\begin{array}{c}\text { Grau de } \\
\text { Relevância }\end{array}$ \\
\hline Categoria do Objetivo & $5,90 \%$ & Estabilidade dos Requisitos & $4,71 \%$ & Tipo de Des vio & $4,11 \%$ \\
\hline Indicador & $5,76 \%$ & Tamanho do Projeto & $4,67 \%$ & Nível & $4,07 \%$ \\
\hline Cliente & $5,27 \%$ & Inovação Tecnológica & $4,63 \%$ & Restrição de Cronograma & $4,05 \%$ \\
\hline Experiência da Equipe & $5,26 \%$ & Tecnologias & $4,47 \%$ & Tamanho da Equipe & $3,89 \%$ \\
\hline Experiência do Gerente & $4,98 \%$ & Domínio da Aplicação & $4,36 \%$ & Duração do Projeto & $3,87 \%$ \\
\hline Natureza do projeto & $4,83 \%$ & Restrição de Recursos Humanos & $4,27 \%$ & Modelo de Ciclo de Vida & $3,66 \%$ \\
\hline Complexidade do Software & $4,79 \%$ & Restrição de Recursos Financeiros & $4,27 \%$ & Paradigma & $3,47 \%$ \\
\hline Tipo de Software & $4,71 \%$ & - & - & & - \\
\hline
\end{tabular}


$\mathrm{Na}$ estratégia para recomendação de ações corretivas, esse conjunto de características é usado para analisar a similaridade entre cenários de ocorrência de desvios. Cada característica é considerada um índice do caso e o grau de relevância de cada característica é interpretado como o peso do índice, conforme descrito a seguir.

Para medir a similaridade entre casos, a estratégia proposta aplica uma medida baseada na medida de similaridade Weighted Nearest Neighbor - WNN, considerando a representação do caso definida para a estratégia. Essa medida calcula a similaridade entre dois casos considerando a soma ponderada da similaridade entre cada índice (cada índice possui um peso a ser considerado no cálculo). A medida de similaridade proposta, denominada SimCOD - Similaridade entre Cenários de Ocorrência de Desvio, indica a grau de similaridade entre dois cenários de ocorrência de desvio: o cenário de ocorrência do desvio atual - CODa e o cenário de ocorrência de um desvio ocorrido no passado - CODp. Detalhes dessa medida, as fórmulas de cálculo e uma situação hipotética ilustrando o uso da medida proposta para análise de similaridade entre dois cenários de ocorrência de desvios são apresentados em (Barreto e Rocha 2010a).

A estratégia proposta foi implementada em um Sistema de Recomendação de Ações Corretivas. Nesse sistema, a alimentação da base de casos se dá automaticamente, sempre que um desvio é detectado, uma ação é executada para tratar esse desvio e o efeito da ação executada é analisado pelo sistema. A Figura 9 mostra a tela do sistema com as ações recomendadas para tratar um desvio detectado, permitindo o registro da ação executada e o prazo esperado para observar o efeito dessa ação.

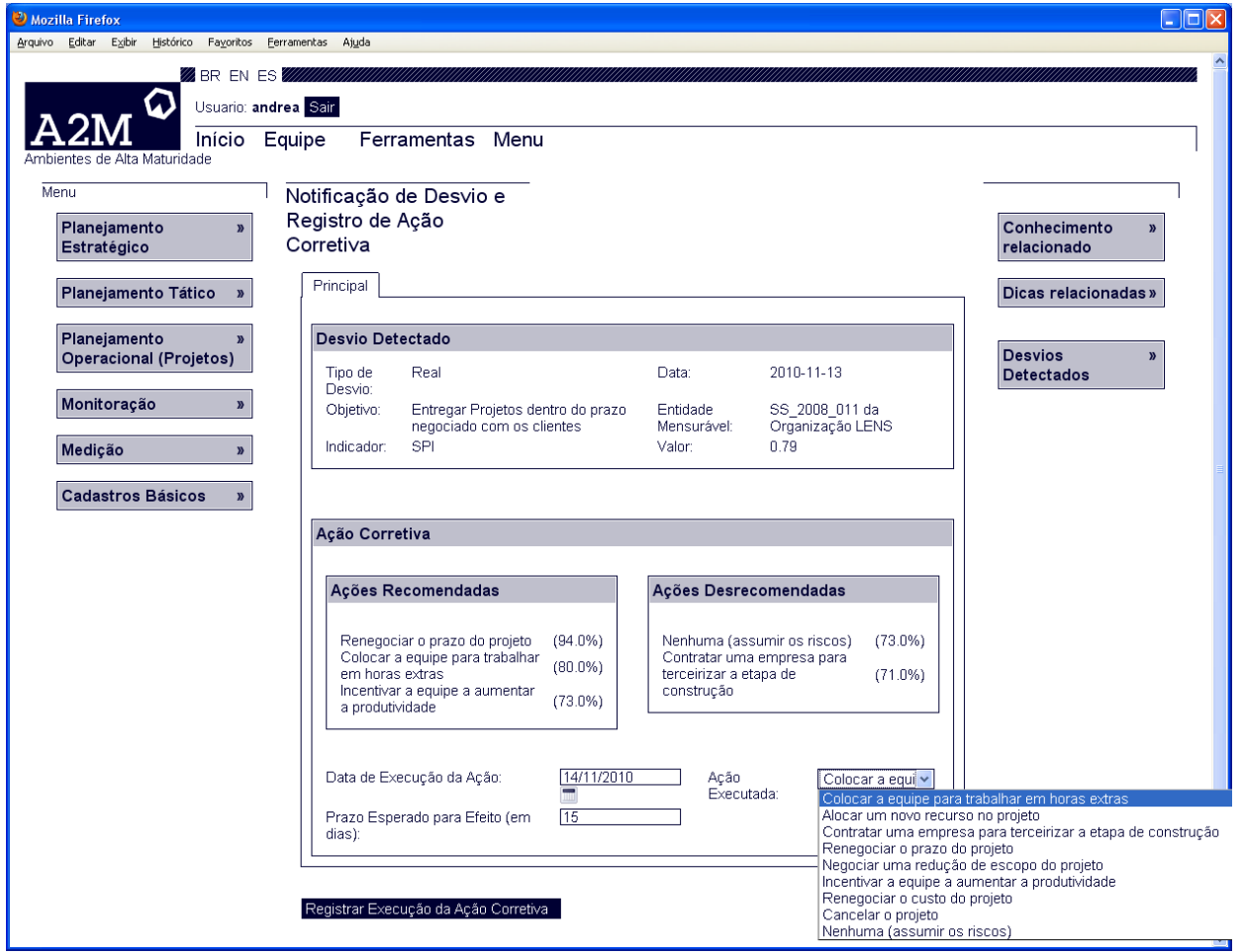

Figura 9. Recomendação de ações corretivas no sistema de recomendação

Para viabilizar a análise automática do efeito das ações executadas e a recomendação das ações mais adequadas dois agentes foram implementados: o agente 
de análise do efeito e o agente de recomendação de ações. $\mathrm{O}$ agente de recomendação implementa algoritmos de recuperação sequencial e de dois níveis. $\mathrm{O}$ agente de análise do efeito da execução da ação é responsável por monitorar continuamente a base de medidas à procura de novas coletas registradas para indicadores associados a desvios com ação executada e ainda sem efeito registrado. Para isso ele verifica um conjunto de heurísticas propostas neste trabalho para a análise desse efeito, identifica aquela que é aplicável a cada coleta e então infere e registra o efeito na base de casos.

\section{Avaliações da Abordagem Proposta}

A abordagem proposta neste trabalho apoia as organizações na definição de objetivos de software alinhados estrategicamente, na execução de ações mais adequadas para tratar desvios relacionados aos objetivos definidos e, consequentemente, no alinhamento estratégico das melhorias de software. Assim, para avaliar a abordagem como um todo seria necessário avaliar o uso de toda a abordagem em organizações de software, desde a elaboração do planejamento estratégico e das melhorias de software alinhadas estrategicamente até a execução de vários projetos que operacionalizassem o planejamento estratégico, de modo a analisar a real contribuição da utilização da abordagem. Nesse contexto, considerando a limitação de tempo, avaliações intermediárias dos componentes da abordagem foram realizadas ao longo deste trabalho, à medida que os componentes foram desenvolvidos. Os resultados obtidos nas avaliações intermediárias vão ao encontro dos resultados esperados para este trabalho.

Com o intuito de analisar a viabilidade de uso do Método para Planejamento Estratégico, Tático e Operacional em Organizações de Software, o modelo e o método propostos neste trabalho foram utilizados em uma organização. Com relação ao método utilizado, na percepção dos participantes a sequência dos passos se mostrou adequada. Segundo os participantes, o uso de um método para orientar as etapas e um modelo de informação para identificar as informações que deveriam ser descritas se mostrou bastante interessante, pois facilitou o planejamento estratégico, guiando as discussões e evitando longas reuniões para se chegar às definições necessárias. Foram identificadas várias melhorias, tanto no método como no modelo de informação propostos, que foram analisadas e implementadas, permitindo uma evolução da proposta. Uma descrição detalhada da aplicação do método proposto pode ser encontrada em (Barreto e Rocha 2009). Os resultados obtidos com essa experiência de aplicação do método apresentam indícios de que a utilização do método orienta as etapas dos planejamentos estratégico, tático e operacional, promovendo a definição de objetivos de melhoria de software alinhados ao negócio da organização.

Em relação à Infraestrutura para Monitoração de Objetivos Estratégicos, Táticos e Operacionais em Organizações de Software, ao longo do desenvolvimento dessa proposta foram realizadas experiências de aplicação dessa infraestrutura no contexto de duas organizações de software. Essas experiências utilizaram um processo off-line, uma vez que, devido a limitações para disponibilizar o ambiente desenvolvido no contexto real das duas organizações, optou-se por solicitar que as organizações enviassem ao pesquisador as informações dos objetivos definidos e das medidas coletadas durante a monitoração desses objetivos. A partir disso, essas informações foram submetidas ao ambiente de monitoração desenvolvido, para que os desvios, potenciais ou reais, fossem detectados. Foram considerados 3 indicadores distintos, 
monitorados em 10 projetos através de 94 coletas. Essas informações permitiram que a infraestrutura proposta detectasse, armazenasse e notificasse 40 desvios. Essas experiências permitiram avaliar se o ambiente de monitoração desenvolvido realmente era capaz de detectar os desvios conforme esperado. Foi possível observar que para todas as coletas registradas no ambiente, os desvios foram detectados corretamente, tanto desvios potenciais como desvios reais. Essas também experiências permitiram identificar melhorias na infraestrutura que direcionaram uma evolução da proposta. As melhorias identificadas foram analisadas e implementadas, dando origem à versão da infraestrutura apresentada. Os resultados obtidos indicam que a aplicação dessa infraestrutura em um contexto real é viável e que tende a auxiliar a detecção contínua e proativa dos desvios.

Para caracterizar a viabilidade de utilização da Estratégia para Recomendação de Ações Corretivas, um estudo experimental foi realizado. A versão inicial da estratégia para recomendação de ações considerava somente a aplicação da técnica de recuperação sequencial e, por isso, inicialmente o sistema de recomendação foi avaliado considerando somente essa técnica. Porém, os resultados obtidos inicialmente indicaram a necessidade de melhorar o desempenho da estratégia com relação à qualidade da recomendação. Por isso, a estratégia para recomendação de ações corretivas e o sistema de recomendação desenvolvido foram evoluídos para também considerarem a aplicação da técnica de recuperação de dois níveis.

O objetivo do estudo foi analisar a estratégia com o propósito de caracterizar a viabilidade de uso com relação ao desempenho (qualidade da recomendação e tempo gasto) do sistema de recomendação que implementa a estratégia proposta em comparação com a opinião de especialistas. Para definir como a qualidade da recomendação seria caracterizada, um estudo baseado em revisão sistemática da literatura foi executado, e com base nesse estudo, as seguintes medidas foram selecionadas: Precisão (probabilidade de uma ação recomendada pelo sistema de recomendação ser adequada), Cobertura (probabilidade de uma ação adequada ser recomendada pelo sistema de recomendação) e F1 (média balanceada entre Precisão e Cobertura). Uma medida de Acurácia definida na literatura também foi selecionada, como uma análise complementar.

Para determinar o que seria um desempenho aceitável quanto à qualidade da recomendação foi considerado que as recomendações dos participantes foram adequadas, uma vez que os participantes são especialistas em gerência de projetos. Assim, os valores das medidas Precisão, Cobertura, F1 e Acurácia para o sistema de recomendação foram calculados com relação à opinião dos participantes. Considerando que as medidas Precisão e Cobertura são conflitantes entre si e que a medida F1 apresenta uma média harmônica dessas duas medidas, a análise da qualidade da recomendação foi baseada somente na medida F1. Para definir o que seria um valor aceitável para a medida $\mathrm{F}$ 1, um segundo estudo baseado em revisão sistemática da literatura foi executado. Esse estudo teve como resultado principal um conjunto de avaliações recentes de sistemas de recomendação usando as medidas Precisão, Cobertura e F1 com valores médios de 60,16\%, 61,12\% e 57,91\%, respectivamente. Assim, foi definido o valor de referência para a medida F1: 57,91\%. Esse valor indica que na média, balanceando a Precisão e a Cobertura, 57,91\% das ações recomendadas 
pelo sistema são adequadas. No estudo realizado neste trabalho, essas medidas foram calculadas para três valores mínimos de similaridade: $60 \%, 65 \%$ e $70 \%$.

Foram definidas duas hipóteses nulas: H01: O sistema de recomendação que implementa a estratégia proposta apresenta valores para a medida F1 inferiores a 57,91\%; e H02: O tempo gasto pelo sistema de recomendação para recomendar as ações mais adequadas é superior ao tempo gasto pelos participantes do estudo para determinar as ações mais adequadas. Os principais resultados do estudo são exibidos na Figura 10.
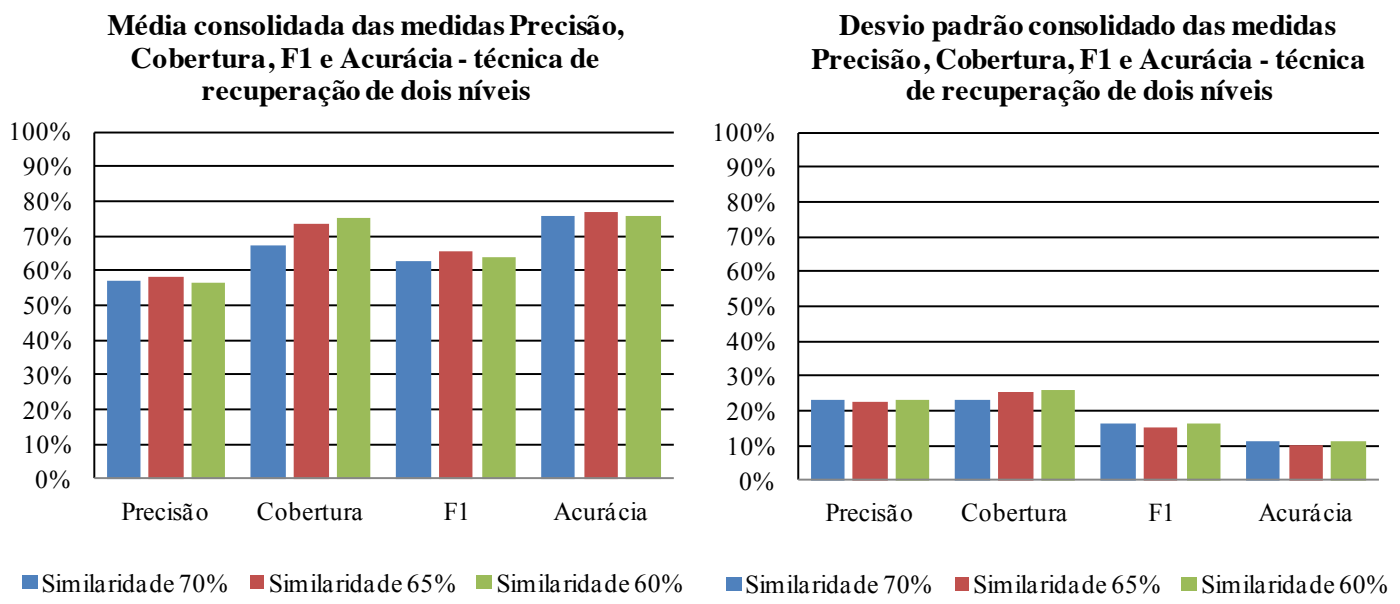

Figura 10. Média e desvio padrão das medidas Precisão, Cobertura, F1, Acurácia

Considerando que o tempo médio gasto pelo sistema de recomendação, quando aplicada a técnica de recuperação de dois níveis, foi de 27 segundos, e que o tempo médio gasto pelos participantes foi de 11,8 minutos, a hipótese nula H02 foi rejeitada. Com relação à qualidade da recomendação, considerando que o sistema, quando aplicada a técnica de recuperação de dois níveis, apresentou valores para a medida F1 superiores a $57,91 \%$, pode-se dizer que o sistema de recomendação apresentou um desempenho aceitável dado que o valor médio da medida F1 (64,01\%) é superior ao valor de referência. Porém, considerando o desvio padrão médio da medida F1 obtida pelo sistema de recomendação $(15,89 \%)$ e o desvio padrão médio do valor de referência $(17,34 \%)$, não foi possível rejeitar a hipótese nula H01. Assim, os resultados deste estudo apresentam indícios de que o desempenho do sistema de recomendação proposto é equivalente ao desempenho dos sistemas considerados no cálculo do valor de referência. A análise complementar da medida Acurácia também mostrou um bom desempenho do sistema de recomendação, uma vez que quando aplicada a técnica de recuperação de dois níveis, a Acurácia foi superior a 75\%. Essa medida indica que, considerando os dados analisados nesse estudo, a probabilidade de a estratégia proposta fazer recomendações adequadas, isto é, recomendar ações adequadas e não recomendar ações não adequadas é superior a $75 \%$. Portanto, os resultados da avaliação experimental apresentam indícios de que a estratégia proposta é útil para o gerente de projetos no momento da monitoração dos objetivos do projeto, uma vez que ela é capaz de fazer boas recomendações de ações para tratar os desvios detectados. Maiores detalhes desse estudo experimental e dos estudos baseados em revisão sistemática da literatura realizados neste trabalho estão descritos em (Barreto 2011). 


\section{Conclusão}

Este trabalho apresentou uma abordagem que apoia as organizações na definição de objetivos de melhoria de software alinhados ao negócio da organização e na gerência desses objetivos de modo a promover a monitoração contínua e a execução de ações mais adequadas para tratar os desvios detectados, considerando, inclusive o cenário das organizações de software de alta maturidade. A abordagem define um modelo de informação e um método para planejamento estratégico, tático e operacional em organizações de software; uma infraestrutura para a monitoração contínua e proativa dos objetivos definidos; uma estratégia para recomendação de ações para tratar desvios detectados na monitoração dos objetivos, e também disponibiliza ferramental de apoio para a utilização da abordagem proposta.

As principais contribuições deste trabalho são: (i) a abordagem para definição e gerência de objetivos de software alinhados ao planejamento estratégico, considerando, inclusive, o cenário de organizações de software de alta maturidade; (ii) o método e o modelo de informação para planejamento estratégico, tático e operacional; (iii) a infraestrutura de apoio à monitoração contínua e proativa dos objetivos; (iv) a estratégia para recomendação de ações corretivas a partir de um aprendizado contínuo sobre a execução de ações na organização; (v) a ferramenta de apoio à execução do método proposto; (vi) o ambiente de apoio à monitoração dos objetivos; (vii) o sistema de recomendação que implementa a estratégia para recomendação; (viii) o conjunto de características relevantes para a análise de similaridade entre cenários de desvio em projetos e o grau de relevância de cada característica; (ix) a medida de similaridade definida $(\operatorname{Sim} C O D)$ que indica a grau de similaridade entre dois cenários de desvio; e (x) a lista de avaliações de sistemas de recomendação e os valores obtidos para as medidas Precisão, Cobertura e F1 resultantes do estudo baseado em revisão sistemática. Esses resultados estão descritos em publicações, conforme citado anteriormente.

\section{Referências}

Appolinario, F., 2006, Metodologia da Ciência: Filosofia e prática da pesquisa, São Paulo, Thomson.

Asato, R., Spinola, M.M., Costa, I., Silva, W.H.F., 2009, "Alignment Between the Business Strategy and the Software Processes Improvement: A Roadmap for the Implementation", In: Portland International Conference on Management of Engineering \& Technology, pp.1066-1071, Portland, Estados Unidos.

Barreto, A.O.S, 2011, Definição e Gerência de Objetivos de Software Alinhados ao Planejamento Estratégico, Tese de D. Sc., COPPE/UFRJ.

Barreto, A.O.S., Rocha, A.R., 2009, "Definição e Monitoração de Objetivos de Software Alinhados ao Planejamento Estratégico". In: VIII Simpósio Brasileiro de Qualidade de Software, Ouro Preto, Brasil, pp. 114-128, Junho.

Barreto, A.O.S., Rocha, A.R., 2010a, "Analyzing the Similarity among Software Projects to Improve Software Project Monitoring Processes", In: 7th International Conference on the Quality of Information and Communications Technology (QUATIC), Porto, Portugal, pp. 441-446, Outubro. 
Barreto, A.O.S., Rocha, A.R., 2010b, "Defining and Monitoring Strategically Aligned Software Improvement Goals", In: 11th International Conference on Product Focused Software Process Improvement, LNCS, v. 6156/2010, Ireland, pp. 380-394.

Becker, A.L., 2007, Alinhamento Estratégico de Programas de Melhoria do Processo de Software em Micro e Pequenas Empresas, Dissertação de M. Sc., PUCRS.

Becker, A.L., Prikladnicki, R., Audy, J.L.N., 2008, "Strategic Alignment of Software Process Improvement Programs Using QFD", In: $1^{\text {st }}$ International Workshop on Business Impact of Process Improvements, pp. 9-14, Leipzig, Germany, May.

Boffoli, N., Bruno, G., Caivano, D., Mastelloni, G., 2008, "Statistical Process Control for Software: a Systematic Approach", In: $2^{\text {nd }}$ IEEE International Symposium on Empirical Software Engineering and Measurement, pp. 327-329, Germany, October.

Bomfim, E., Oliveira, J., Souza, J.M., 2007, "Using a CBR Approach Based on Ontologies for Recommendation and Reuse of Educational Processes", International Journal of Web Based Communities, v. 3, n. 2, pp. 170-182.

Chiavenato, I., 2000, Administração: Teoria, Processo e Prática, SP, MakronBooks.

Guzmán, J.G., Mitre, H.A., Amescua, A., Velasco, M., 2010, "Integration of strategic management, process improvement and quantitative measurement for managing the competitiveness of software engineering organizations", Software Quality Journal, v. 18 , n. 3, pp. 341-359.

McBride, T., 2008, "The mechanisms of project management of software development", Journal of Systems and Software, v. 81, n. 12, pp. 2386-2395.

Montoni, M.A., 2010, Uma Investigação sobre os Fatores Críticos de Sucesso em Iniciativas de Melhoria de Processos de Software, Tese de D. Sc., COPPE/UFRJ.

Pries-Heje, J., Johansen, J., 2010, SPI Manifesto, Version A.1.2.2010.

Robillard, M.P., Walker, R.J., Zimmermann, T., 2010, "Recommendation Systems for Software Engineering", IEEE Software, v. 27, pp. 80-86.

Santos, V.A., Cortés, M.I., 2010, "Organizational Knowledge Management through Software Process Reuse and Case-based Reasoning", In: 12th International Conference on Enterprise Information Systems, pp. 223-228, Madeira, Portugal.

SEI, 2010, Capability Maturity Model Integration (CMMI) for Development, Version 1.3, Carnegie Mellon University, Software Engineering Institute.

SOFTEX, 2009, MPS.BR - Melhoria de Processo do Software Brasileiro, Guia Geral : 2009, SOFTEX - Associação para Promoção da Excelência do Software Brasileiro.

Thies, A., Roth, C., 2010, "Recommending Rename Refactorings", International Workshop Recommendation Systems for Software Engineering, pp.1-5, South Africa.

Trindade, C.C., 2009, Presley: Uma Ferramenta de Recomendação de Especialistas para Apoio à Colaboração em Desenvolvimento Distribuído de Software, Dissertação de M. Sc., Centro de Informática, UFPE, Recife, Brasil.

Wangenheim C.G., Wangenheim, A., 2003, Raciocínio Baseado em Casos, Manole, SP.

Wazlawick R.S. 2009, Metodologia de Pesquisa para Ciência da Computação, Elsevier 